\title{
Neck Irradiation, Carotid Stenosis and Carotid Intima-Media Thickness as Stroke Predictor
}

\author{
Garima Khatri ${ }^{1}$, Mohan Singh $^{1}$, Sunita Bika ${ }^{2}$, B. L. Mehta ${ }^{1}$ \\ ${ }^{I}$ (Department of Anatomy, S. P. Medical College, Bikaner, Rajasthan, India) \\ 2. (Department of Pathology, S. P. Medical College, Bikaner, Rajasthan, India)
}

\begin{abstract}
Carotid stenosis is a major sequela of head and neck irradiation that has not received the attention it deserves. Its impact on the quality of life of patients can be substantial. This review discusses the incidence of radiation induced carotid stenosis and its role in prediction of stroke. Atherosclerotic changes of the common carotid artery(CCA) may appear like irregularities of the intima-media thickness (IMT) in B-mode ultrasound. Early detection can help to decrease or slow down the rate of prognosis of atherosclerosis and this can improve the quality, and increase the length of patient's life. The incidence of significant increase in carotid IMT following head and neck irradiation is seen. Patients with carotid stenosis are at increased risk for stroke. In this study, subjects with any other major risk factor for atherosclerosis and previous history of cerebrovascular accidents were excluded.
\end{abstract}

Key words: Ultrasound, Carotid stenosis, Neck irradiation, Atherosclerosis, Intima-media, cerebrovascular accidents

\section{INTRODUCTION}

Cancer is a dreadful disease, growing at a tremendous rate globally, and has become the second leading cause of death in the United States. Even more agonizing than the mortality rate is the emotional and physical suffering inflicted by neoplasm. About 60 percent of all cancer patients require radiation therapy as the part of their care. Radiotherapy as a single modality or in combination with surgery has been widely used in the treatment of head and neck tumors and this has resulted in marked improvement in survival of patients with these tumors. Successful treatment increases survival but also puts the patient at the risk of radiation related side effects. Of these, vascular side effects are serious and may be life threatening. . Post-radiation injury to the large vessel has not received as much attention as micro vascular irradiation injury. Ischemic stroke is one of the most important determinants of late morbidity and mortality after radiotherapy. The magnitude of a possibly increased risk of future stroke due to radiotherapy to the neck cannot be extracted from the data and cases published so far. Evidence for an increased risk of stroke was provided in a cohort study by Elerding et al, [1] but they were not able to establish a definite relation between RT on the neck and increased risk of stroke.

Change in IMT of the carotid artery has become well established in epidemiological studies, as a marker of the early changes of atherosclerosis. Atherosclerosis associated with radiation therapy, while histologically similar to spontaneous atherosclerosis, is clinically distinct because it is limited to the irradiated area and is less likely to be associated with atherogenic risk factors.

RT could lead to enhancement of IMT progression, resulting in carotid stenosis and consequently to cerebrovascular stoke.

So the present study aims to establish the effect of radiations on IMT of common carotid artery in head and neck cancer patients and also to establish that whether IMT of common carotid artery is a predictor of future stroke.

\section{MATERIALS AND METHODS}

\subsection{Study design}

This study was a cross sectional study conducted in the Department of Anatomy in collaboration with the Department of Radiation Oncology, Acharya Tulsi Regional Cancer Research Center and Department of Radiodiagnosis, Sardar Patel Medical College and Associated Group of Hospitals, Bikaner, Rajasthan. It incorporated 271 subjects. 232 were controls and 39 were irradiated patients of head and neck malignancies. All subjects were without any risk factor. The study obtained approval of Hospitals Ethics Committee as well as prior consent of the subjects in the form of an Informed Consent Form.

\subsection{Inclusion criteria for cases}

Patient fulfilled following inclusion criteria:

1. Must have received radiation therapy to the carotid area for treatment of malignancy.

2. Radiotherapy to have occurred at least 1 year before the ultrasound examination. 
3. Should not have other major risk factors for atherosclerosis, such as, diabetes mellitus, hypertension, hypercholesterolemia, obesity, cigarette smoking habit.

4. All patients were treated with Cobalt therapy.

\subsection{Inclusion criteria for controls}

Controls were non- irradiated apparently healthy subjects without any major risk factors for atherosclerosis like, diabetes mellitus, hypertension, hypercholesterolemia, obesity, cigarette smoking habit.

\subsection{Data analysis}

All patients and control subjects completed a questionnaire to assess their cardiovascular risk factors and accidents, had their height, weight and blood pressure measured and provided a blood sample for measurement of total cholesterol and random blood sugar levels. Smoking history was assessed.

Then we evaluated the association between neck irradiation and common carotid intima-media thickness, and also 10 years stroke probability percent by using point system proposed by Framingham Heart Study (FHS) [2].

\subsection{Technique}

\subsubsection{Ultrasound Examination}

All scans were obtained by Color Doppler and B-mode ultrasound technique . B-mode ultrasonography (US) is a diagnostic method which allows measurement of intima-media thickness (IMT) at the level of carotid artery. B-mode ultrasound of carotid arteries is increasingly used to assess the atherosclerotic burden of the arterial system.

All carotid ultrasound scans were carried out by one observer using previously standardized program incorporated in software package of the ultrasound equipment. Examinations were performed after a rest period of $10 \mathrm{~min}$., with subjects in supine position with neck extended Both common carotid arteries were examined along their full visible length.

\subsubsection{IMT Measurements}

Sonological examination of Common Carotid Artery (CCA) was done using L\&T SEQUINA Color Doppler Scanner with a linear band probe of frequency 6.6 to $14 \mathrm{MHz}$. All scans were obtained at $12 \mathrm{MHz}$. All IMT measurements were made in the longitudinal plane at the point of maximum thickness on the far wall of the common carotid artery along a $1 \mathrm{~cm}$ section of the artery proximal to the carotid bulb. The position of the carotid bulb is defined as the point at which the far wall deviates away from the parallel plane of the distal common carotid artery. IMT was the distance between the inner echogenic line representing the intima-blood interface and the outer echogenic line representing the adventitia - media junction. After freezing of the image, the measurement was made with electronic calipers. The ultrasound images were magnified to improve the accuracy of caliper placement. Measurements were repeated three times, unfreezing the image on each occasion. The mean value of each set of three measurements, representing the mean IMT of each common carotid artery was taken. Hard copy of images was obtained of each examination.

\section{OBSERVATIONS}

Table 1: Comparative biometrics of controls and cases

\begin{tabular}{|c|c|c|c|}
\hline Parameters & $\begin{array}{l}\text { Controls }(n=232) \\
(\text { Mean } \pm \text { SD })\end{array}$ & $\begin{array}{l}\text { Cases }(n=39) \\
(\text { Mean } \pm \text { SD) }\end{array}$ & $P$ value \\
\hline Age (yrs) & $42.12 \pm 13.61$ & $48.07 \pm 9.86$ & $0.010 * *$ \\
\hline Height (cm) & $170.93 \pm 7.16$ & $170.46 \pm 6.33$ & $0.70 \mathrm{NS}$ \\
\hline Weight (kg) & $67.13 \pm 8.35$ & $63.71 \pm 6.20$ & $0.016^{* *}$ \\
\hline Total Cholesterol (mg/dl) & $182.88 \pm 23.50$ & $168.55 \pm 13.24$ & $0.0001 * * *$ \\
\hline $\begin{array}{l}\text { Random Blood Sugar } \\
\text { (mg/dl) }\end{array}$ & $93.13 \pm 24.12$ & $97.15 \pm 17.30$ & $0.327 \mathrm{NS}$ \\
\hline $\begin{array}{l}\text { Blood Pressure Systolic } \\
(\mathrm{mmHg})\end{array}$ & $126.92 \pm 7.02$ & $129.61 \pm 8.77$ & $0.036^{*}$ \\
\hline $\begin{array}{l}\text { Blood Pressure Diastolic } \\
\text { (mmHg) }\end{array}$ & $82.22 \pm 2.21$ & $83.07 \pm 2.36$ & $0.03 *$ \\
\hline BMI & $22.95 \pm 1.92$ & $22.09 \pm 1.62$ & $0.009 * *$ \\
\hline Mean IMT (mm) & $0.310 \pm 0.038$ & $0.690 \pm 0.065$ & $0.0001 * * *$ \\
\hline
\end{tabular}


Neck Irradiation, Carotid Stenosis and Carotid Intima-Media Thickness as Stroke Predictor

\begin{tabular}{|l|c|c|c|}
\hline $\begin{array}{l}\text { Total Points for Risk } \\
\text { Factors }\end{array}$ & $2.99 \pm 1.13$ & $3.56 \pm 1.87$ & $0.010^{* *}$ \\
\hline
\end{tabular}

*significant at 5\%, ** significant at 1\%,*** highly significant

TABLE 1 shows comparative biometrics of controls with those of cases. There are various parameters which are compared between controls and cases on the basis of their mean values and the significance of this difference is shown by $\mathrm{p}$ value.

Mean IMT: Mean IMT in controls was much lower in comparison to cases. In controls it was $0.310 \pm$ $0.038 \mathrm{~mm}$ while in cases it was much higher i.e. $0.690 \pm 0.065 \mathrm{~mm}$. The difference was highly significant $(\mathrm{p}<0.001)$.

Total Points for Risk Factors: These were calculated using risk score profile for stroke, put forth by FHS [2]. Its mean value in controls was lower than the cases showing increased risk of stroke in irradiated patients. Its mean value in controls was $2.99 \pm 1.13$ and in cases it was $3.56 \pm 1.87$. The difference was significant i.e. $\mathrm{p}<0.05$.

Analysis of our data documents two major findings:

\subsection{Increase in carotid IMT after radiation}

There is thickening of the intima-media complex of the common carotid artery in patients with a history of radiations to the head and neck region compared with matched non-irradiated subjects, which is clearly indicated in Fig. 1. The Mean IMT of controls and cases was recorded $0.310 \pm 0.038 \mathrm{~mm}$ and $0.690 \pm$ $0.065 \mathrm{~mm}$, respectively which shows that in cases, mean IMT is higher and the difference between the two was highly significant i.e. $\mathrm{p}<0.001$.

Above data indicate clearly that patients who have radiotherapy for the treatment of head and neck malignancy show higher carotid IMT and are at increased risk of accelerated atherosclerosis in common carotid arteries. Therefore, the findings from non-invasive imaging investigations support the important role of radiation exposure for the development of atherosclerotic changes.

\subsection{IMT of common carotid artery is a good predictor of stroke}

The probability of stroke may be determined by using a point system from the Framingham Heart Study [2]. Our study has proposed IMT of CCA as a new parameter to predict 10 years probability percent of stroke.

Our findings show that Mean Total Points for Risk Factors in controls was $2.99 \pm 1.13$ while in cases , it was higher i.e. $3.56 \pm 1.87$. The difference was significant $(\mathrm{p}<0.05)$. This trend of increasing total points for risk factors with increasing carotid IMT is indicated in Fig.2. So it is clear from this study that patients with carotid stenosis are at increased risk of stroke (Abayomi Olubunmi K [3], Chambless L.E. et al. [4], and Bots M.L et al [5]). Our findings are in agreement with previous observations such as that of King LJ et al. [6] who reported that patients irradiated for Hodgkin's Lymphoma manifested a larger number of abnormal ultrasound scans, carotid stenosis and greater IMT than matched controls. Similarly So NMC et al. [7] who compared IMT in patients irradiated for nasophyryngeal carcinoma with a control group and found that the mean carotid IMT was greater in the treated patients $(p<0.05)$. Shariat $M$ et al. [8] documented in their study that the mean IMT in irradiated group measured $0.74 \mathrm{~mm}$ which was higher in comparison to matched controls which showed mean IMT $0.46 \mathrm{~mm}$. The difference was highly significant $(\mathrm{p}<0.001)$.

\section{CONCLUSIONS}

This research broadly gives two conclusions in support of achievement of the aims of this study:

5.1 Common Carotid IMT was increased after radiotherapy to the head and neck region.

5.2 Increased Carotid IMT predicts increased risk of stroke in irradiated patients.

It is advisable to adopt advanced techniques for radiotherapy to screen unnecessary exposure of healthy tissues. As changes in the carotid IMT are the earliest indicators of atherosclerosis, routine ultrasound of the carotid arteries is advisable for patients who have received radiotherapy to the head and neck. Early detection can help to decrease or slow down the rate of prognosis of atherosclerosis and this can improve the quality, and increase the length of patient's life.

Gender wise evaluation of radiation injury to the large vessels needs to be accomplished .Irradiated subjects with risk factors should be incorporated and compared. The exact mechanism of radiation induced injury in the large vessels is not yet well understood. Histologic studies might through some light in this matter. The relationship between radiation dose and large vessel injury needs to be further investigated. The rate of progression of atherosclerosis in irradiated vessel needs further evaluation. 


\section{Acknowledgements}

We gratefully appreciate the technical and statistical analysis support of the concerned departments and subjects who participated in this research. A word of thanks for everyone who encouraged and assisted this work.

\section{REFERENCES}

[1] S.C. Elerding, R. N. Fernandez, J. C. Grotta , R.D. Lindberg, L.C. Causay, M. J. McMurtrey, Carotid artery disease following external cervical irradiation, Ann Surg,. 194 (5 ),1981,609-15.

[2] Framingham Heart Study: www.framinghamheartstudy.org.

[3] K. Abayomi Olubunmi, Neck irradiation, carotid injury and its consequences, Oral Oncol., 40, 2004, 872-8.

[4] L. E. Chambless, A. R. Folsom, L. X. Clegg, et al., Carotid wall thickness is predictive of incident clinical stroke: The Atherosclerosis risk in communities (ARIC) study, Am. J. Epidemiol., 151, 2000, 478-87.

[5] M. L. Bots, A. W. Hoes, A. Hofman, J C M Witteman, D. E. Grobbee, Cross-sectionally assessed carotid intima-media thickness relates to long-term risk of stroke, coronary heart disease and death as estimated by available risk functions, Journal of Internal Medicine, 245, 1999, 269-276.

[6] L. J. King, S. N. Hasnain, J. A. W. Webb, J. E. Kingston, E. A. Shafford, T. A. Lister, J. Shamash, R. H. Reznek, Asymptomatic carotid arterial disease in young patients following neck radiation therapy for hodgkin lymphoma, Radiology, 213, $1999,167-172$.

[7] N. M. C. So, W. W. M.Lam, P. Chook, et al., Carotid intima-media thickness in patients with head and neck irradiation for the treatment of nasopharyngeal carcinoma, Clin. Radiol., 57, 2002, 600-3.

[8] M. Shariat, A. Alias, B. M. Biswal, Radiation effects on the intima media thickness of patients with head and neck malignancy the common carotid artery in post-radiotherapy, Postgrad. Med. J., 84, 2008, 609-612.

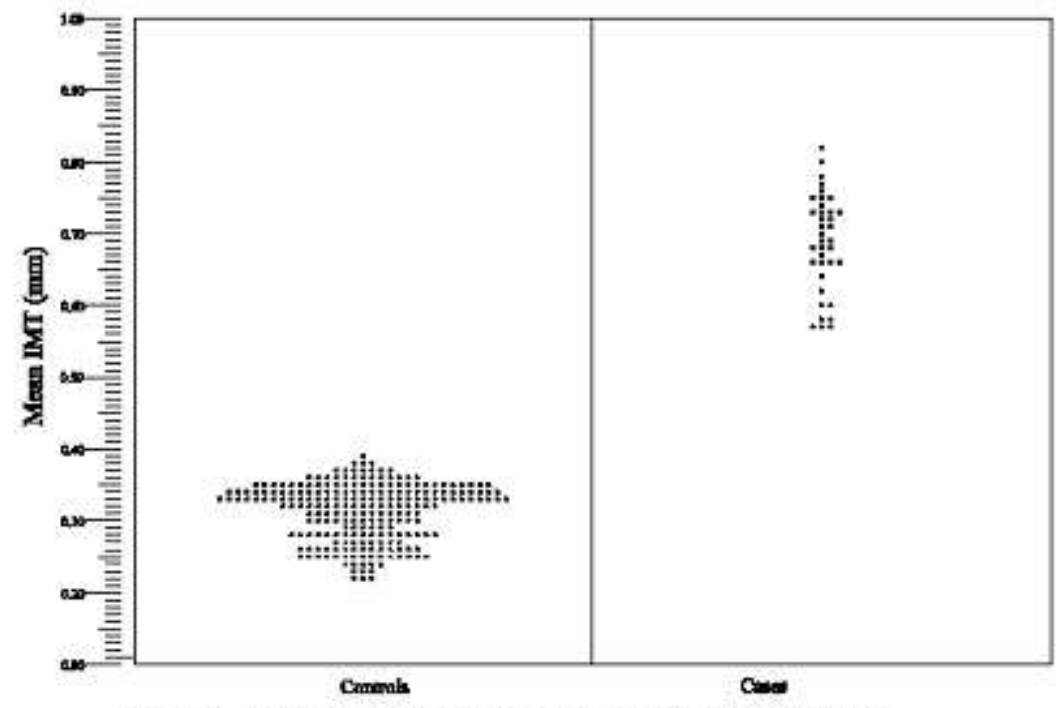

Figure 1. Scatherogram representing Mean IMT in controls and cases

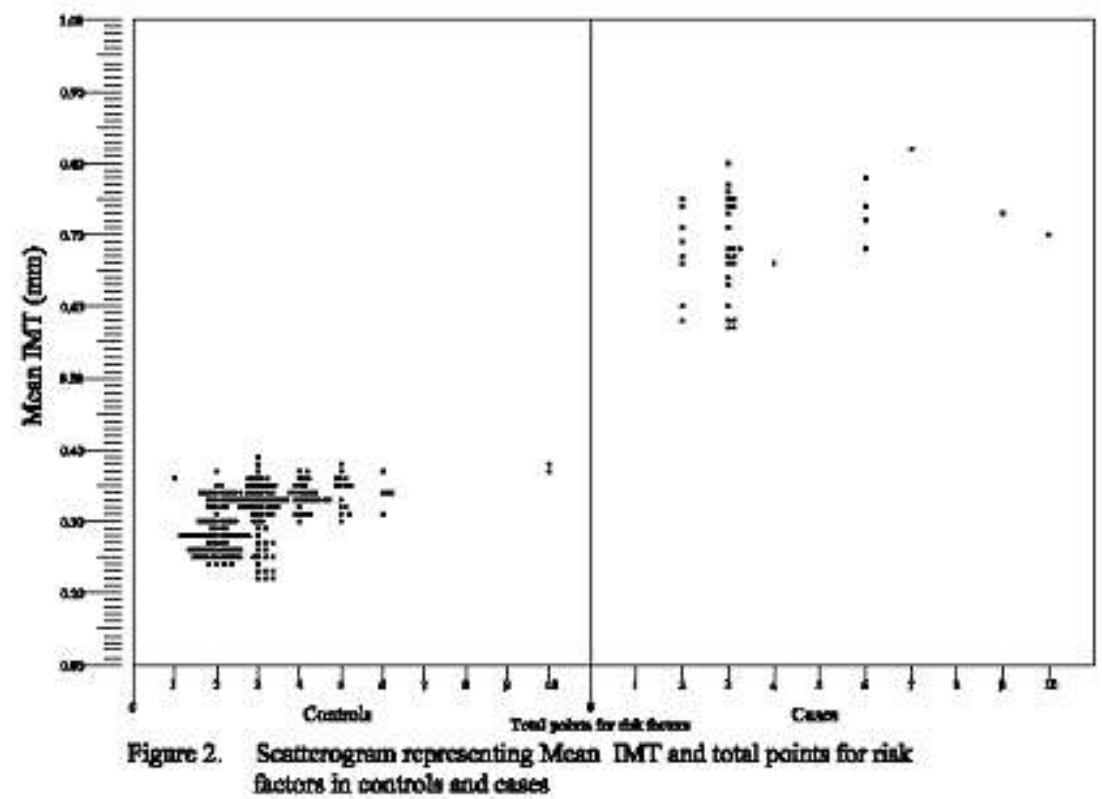

